\title{
La enseñanza en Melilla durante la Dictadura de Primo de Rivera
}

\section{M..$^{a}$ Josefina Garcia Alonso}

\section{1.-INTRODUCCION}

El período que comprende este trabajo transcurre desde la subida al poder de Primo de Rivera (1923) hasta su dimisión en enero de 1930.

La enseñanza en Melilla durante este período es una continuidad de los años precedentes junto a las reformas propias que se llevaron a cabo durante la Dictadura y que afectaron a la ciudad de una manera importante, especialmente en la Segunda Enseñanza.

Analizaremos aquellas actuaciones que tanto a través de la Junta de Arbitrios (formada por militares y civiles) como por la Junta Municipal, creada en el año 1927, cooperaron en el desarrollo de la enseñanza y educación.

España desde 1912 hasta 1956 ejerce sobre una pequeña zona del norte de Africa un régimen de Protectorado y Melilla, dentro de esta zona y como plaza de soberanía, atiende no sólo a intereses españoles, sino también a intereses marroquies e israelitas, minorias representantes de una élite económica y cultural.

A la problemática social, política, que durante estos años fue notable en acontecimientos, hay que añadir el problema económico con características especiales debido al singular régimen aduanero de la ciudad. Esto reportará beneficios a la enseñanza.

La población de Melilla que en 1910 era de 9.000 habitantes, había aumentado a 50.000 en 1920. Ello creó un problema, no sólo para la enseñanza primaria, sino para los estudios de bachillerato. El nacimiento de la población había sido tan rápido que no había dado tiempo suficiente a un proceso meditado y progresivo de creación de centros de enseñanza.

\section{2.-Fuentes documentales y bibliográficas}

Para la documentación del presente trabajo me he servido de las Actas de la Junta de Arbitrios (1923-1927). También ha sido de gran valor documental el periódico local $E l$ Telegrama del $R i f$, que reflej: ios acontecimientos más importantes de la vida ciudadana. Como bibliografía: Un texto editado en el año 1956 que recoge toda la labor de España en Marruecos a través de la Delegación de Educación y Cultura, de Fernando Valderrama Martínez, "Historia de la Acción Cultural de España en Marruecos (1912-1956)", Tetuán: Editora Marroquí, 1956. 
Los Anuarios de los años 1927-28 Anuario-Guia Oficial de Marruecos y del Africa Española.

Ha sido muy escasa la documentación recogida de la enseñanza privada a pesar del elevado número de alumnado que acogía. Sirva de referencia la obra de Saturnino Gallego, "Sembraron con amor. La Salle: Centenario en España (18781978)", San Sebastián, 1978. Como planteamiento de Fondo puede ser útil la obra "Historia de los sistemas educativos" editada por la UNED, Madrid, 1980.

\section{3.-La enseñanza primaria}

\section{1.-Los primeros años}

Consistía en aplicar, en principio, las normas vigentes en España sobre la base de disponer de locales y personal docente.

Debido al aumento de población, fue preciso disponer de más escuelas para atender a la población infantil española, que iba en aumento, sin olvidar que numerosos israelitas deseaban que sus hijos recibieran instrucción en estas escuelas y asimismo algunos musulmanes, aquellos que más en contacto habian estado con España y con su cultura.

\section{2.-Las primeras disposiciones}

Una R. O. del Ministerio de Estado de 24 de febrero de 1923, señala una preocupación, por ordenar el sistema orgánico de la instrucción elemental española, en la zona del Protectorado de Marruecos. Para lo que se crean determinadas escuelas graduadas y unitarias. Se reglamenta la situación de los maestros españoles en ejercicio, asi como los que ingresan en virtud del primer concurso examen, que se convocaba para cubrir las plazas de nueva creación.

En el B. O. de 15 de octubre de 1929, fue publicado el primer escalafón de maestros, maestras y profesores especiales.

Por R. O. de 19 de febrero de 1925, se organizó en régimen de Grupo Escolar la enseñanza primaria, bajo la gestión de un Director por cada Grupo. Este sería designado libremente por la Administración entre los maestros y maestras de los respectivos centros.

\section{3.-El Estatuto}

Ya en el año 1924 hubo un plan de estatuto para la enseñanza primaria española, que no llegó a concretarse. La tarea verdadera se inicia en 1929 con el Inspec- 
tor don Alfonso Barea (1), que redactó un proyecto que constaba de 183 títulos.

No fue este, sin embargo, el definitivo. Un nuevo esquema fue sometido a una ponencia de maestros que, representando las distintas ciudades y regiones, se reunió en Tetuán bajo la presidencia del Inspector don Manuel Chacón (2) dando redacción definitiva al "Estatuto de la Primera Enseñanza Española de la Zona", fue publicado en noviembre de 1930.

Esta reglamentación, perfectamente concebida de acuerdo con el estado de la enseñanza en aquellos años, comprendia los siguientes capitulos en 137 artículos: I "De los escolares"; II "De la enseñanza"; III "De las escuelas"; IV "Del Magisterio"; V "De la Inspección"; VI "De los edificios y del material".

Aunque el Estatuto ha sufrido modificaciones a través de los años su importancia fue extraordinaria hasta el punto de que toda la legislación posterior en materia de organización escolar ha tenido como base el Estatuto que comentamos.

\section{4. - La Inspección}

La Inspección de Primera Enseñanza de Melilla, en principio, pertenecía a la circunscripción de la primera zona de la provincia de Málaga.

Su misión consistía en velar por la enseñanza y aconsejar en materia educativa. Estaba en contacto permanente con la Junta de Arbitrios, más tarde Junta Municipal, para proponer a ésta la creación o ampliación de escuelas o grupos escolares cuando la necesidad lo exigiese; y que las escuelas tuviesen el material escolar indispensable.

\section{5.-La Junta de Arbitrios y las escuelas}

Los maestros de instrucción pública aparecen como empleados municipales aunque su nombramiento sea por parte del Ministerio. La Junta tenía competencia para nombrar a maestros interinos. Asimismo se crea una Comisión compuesta por señores Vocales para que todos los niños de la ciudad puedan recibir instrucción y para indicar los lugares donde sea preciso llevar a cabo las construcciones escolares necesarias.

Es de resaltar la suma considerable que la Junta empleó, tanto en nuevas construcciones, como en ampliaciones, durante el período de la Dictadura. Los acontecimientos de carácter político supusieron una importante recuperación social. Ello

(1) Don Alfonso Barea Molina. Director interino de la E. General y Técnica de Melilla, 27 de noviembre de 1926 por R. O. de la Presidencia del Consejo de Ministros, Dirección General de Marrue$\cos$ y Colonias.

(2) Don Manuel Chacón y Sánchez, Catedrático por oposición de Psicología y Lógica y Secretario General de la E. Central y Técnica de Melilla. 
unido al carácter militarista de la ciudad, tanto por la guarnición de tropas que albergaba como por los componentes de la Junta de Arbitrios. El Presidente era un general de Brigada, y numerosos vocales, militares de alta graduación formaban parte de ella, asi como un teniente vicario.

El censo escolar de Melilla recogido en el año 1924 se acerca a 10.000 niños. Funcionan 35 escuelas y faltan muchas más. Hay muchos niños que no van a la escuela. Los datos al efecto recogidos son:

Existen funcionando 25 escuelas públicas y 10 privadas. Respecto a las primeras, son las siguientes:

De niños, ocho, instaladas:

Dos en Ataque Seco; una en el Barrio Obrero; una en el Barrio del General Arizón; una en el Barrio del Real; una en el Barrio de Alfonso XIII.

De niñas, nueve, instaladas:

Una en la parte alta de la ciudad; tres en el Barrio de Alfonso XIII; una en el Hipódromo; dos en el Barrio del Real.

De párvulos, ocho, instaladas:

Dos en la parte alta de la ciudad; tres en Ataque Seco; una en el Barrio de Alfonso XIII; una en el Hipódromo; dos en el Barrio del Real.

A las escuelas de niños asisten aproximadamente unos 389 niños, siendo la matrícula de ellos de 508 .

A las de niñas concurren 522, siendo la matrícula de 604. En las de párvulos van a clase 432, ascendiendo la matrícula a 502. En total asisten a unas y otras escuelas 1.733 niños.

Siendo el censo escolar, el último confeccionado, de 9.834 niños, incluidos los párvulos y excluidos los niños mayores de 12 años -hasta 14 deberían figurar con arreglo al Estatuto del Magisterio- y estando dispuesto que a cada centro de enseñanza deben asistir 60 niños como máximo, resulta que el número de escuelas que corresponde a Melilla es el de 163. Como existen funcionando 35, entre públicas y privadas, faltan 129.

A las 10 escuelas no públicas asisten, según cálculos aproximados, unos 1.500 niños. Es decir, que reciben instrucción unos 3.000 escolares.

Quedan más de 6.000 niños que no asisten a las escuelas. Sólo parte de la población quedaba prácticamente atendida, la burguesía. Otros sectores, la clase obrera, los barrios periféricos, quedaban sin recibir la escolarización necesaria. Las causas hay que buscarlas, no necesariamente en la Junta de Arbitrios, que siempre atendió económicamente a los posibles problemas. Tenemos que pensar en que la sociedad no estaba todavía demasiado sensibilizada hacia la instrucción, y también, que muchos niños en edad escolar estaban realizando trabajos permitidos legalmente. La obligatoriedad de la enseñanza, por tanto, estaba sujeta a conceptos de tipo moral, de rentabilidad social, de reconocimiento hacia la dignidad de la persona, etc.

Respecto a la escolaridad obligatoria aparecen leyes al respecto en:

Manifiesto de Primo de Rivera (1923); Decreto de 20 de octubre de 1923; Anteproyecto de la Constitución de la Monarquía (1929). Artículo 22: 
"Todos los españoles están obligados:

...

3.') A dar a los hijos que tuvieren y a los menores confiados legalmente a su cuidado, la instrucción elemental por los medios a su alcance, o haciendo que asistan a la Escuela Primaria Pública."

\section{6.- La Enseñanza Primaria israelita}

La población israelita utiliza normalmente el español como lengua materna. Acuden los niños a las escuelas españolas participando con sus compañeros en todas las tareas escolares, excepción hecha de la clase de religión.

En noviembre de 1926, la Comunidad israelita solicita a la Junta de Arbitrios, la apertura de una escuela de niños y otra de niñas, en un edificio de su propiedad, con profesores españoles. Una mayoria aprobó su apertura nombrando dos maestros para tal misión. Funcionan igual que cualquier escuela oficial del Estado español excepto la religión, para lo cual la Colonia israelita nombra profesores especiales. Su enseñanza se rige por el calendario religioso.

\section{7.-La Escuela indígena}

Su creación se debe al gobernador militar de Melilla en el año 1907, el general Marina, y la cooperación de la Cámara de Comercio que lo solicitó del Gobierno.

Viene funcionando este centro con una matrícula de 50 niños, 10 de los cuales son de Farhana y los restantes de la Plaza. Las clases se hallan divididas en tres grados, de iniciación, primero y segundo. Son gratuitas.

En junio del 1924 en la Escuela General y Técnica se inaugura un local destinado a internado de niños indígenas. Asisten, en principio 10 niños, hijos de algunos notables de las diversas cabilas sometidas. La enseñanza está a cargo de profesores europeos e indigenas. En el internado se respeta la religión, uso y costumbres de los nuevos alumnos.

\section{8. - Las escuelas del Ave María}

Dentro de la Enseñanza Primaria merece especial mención la creación de las escuelas del Ave María. Su "acción social" fue muy importante porque acogió a un abundante número de niños de ambos sexos, que estan sin escolarizar, debido a la ausencia de escuelas nacionales en el lugar donde se ubicaron, Barrio de Bateria J., acogiendo también a niños de los barrios Príncipe de Asturias y Reina Regente.

Su fundación se debe a un Patronato de Acción Social Popular formado por el vicario eclesiástico señor Carasola y civiles. Cuenta con el apoyo económico de la Junta de Arbitrios, que proporcionó en primer lugar un espacioso lugar. Coopera- 
ban en su mantenimiento diversas entidades de Melilla y de particulares con sus donativos y suscripciones mensuales. Fue nombrado un maestro por parte del Ministerio de Instrucción Pública. Se hicieron necesarias sucesivas ampliaciones debido al gran número de alumnos que concurrian.

\section{9.-La acción social y la educación}

Las escuelas obreras del Buen Pastor regidas por las Hermanas del Buen Pastor, estaban establecidas en el colegio de las hermanas del Buen Consejo. Su misión era la enseñanza a jóvenes obreros que concurrían a dicho centro fuera de las horas de trabajo. La matrícula era de 115 alumnos.

\subsection{0-La Protección escolar}

Las cantinas escolares establecidas en diferentes barrios y sufragadas por la Junta de Arbitrios con la ayuda de la Asociación General de Caridad realizaron una importante acción social.

\subsection{1-La escuela de ciegos}

A instancia de don Enrique Millet se crea una escuela de ciegos en un local facilitado por la Junta. La enseñanza era gratis. Concurren a dichas clases 18 ciegos. La remuneración corría a cargo de la Junta.

Al finalizar el periodo que analizamos la creación de centros escolares atendido por profesionales se habia duplicado.

\section{4.-LA ENSENANANZA MEDIA}

Hasta el año 1920 Comisiones de catedráticos de los institutos de Málaga o de Almería se desplazaban a Melilla para examinar a los alumnos que realizaban estudios de enseñanza media.

Una comisión integrada por personalidades de la ciudad, cristianos, musulmanes e israelitas se dirigió a Madrid para visitar al Ministro de Instrucción Pública. El resultado fue la creación de la Escuela General y Técnica.

Por Real Decreto de 14 de junio de 1921 fue creado un Instituto, que otro Real Decreto de 31 de agosto de 1922 convirtió en Escuela General y Técnica con lo siguientes estudios:

Bachillerato; Magisterio; Estudios mercantiles; Artes y Oficios; Enseñanzas industriales.

La escuela pasó a denominarse Instituto General y Técnico “Victoria Euge- 
nia", en el año 1930 con motivo de la visita de S.S. M.M. Continuó con el mismo régimen hasta el año 1932, en que recibe el nombre de Instituto Nacional de Enseñanza Media, uniéndose al régimen de los de su clase en España y separándose de él el resto de las enseñanzas para pasar a constituir centros independientes, siendo el primero la Escuela Normal.

Tanto el Instituto, en sus dos épocas, como la Escuela Normal y los demás centros de enseñanza han ejercido una considerable influencia sobre las poblaciones musulmanas e israelita, siendo numerosos los alumnos que han pasado por sus aulas.

\section{1.-La Escuela General y Técnica}

La originalidad que ofrecia el proyecto de Real Decreto de la Escuela en la legislación vigente de Instrucción Pública, era la de fusionar cuatro secciones, que constituyen en España cinco centros separados y autónomos.

El proyecto suponía obtener de los presupuestos generales del Estado 100.000 pesetas de subvención y una organización que se apartaba de las instituidas en el Ministerio desde el primer momento.

El proyecto era toda una reforma de economía para la enseñanza especial y secundaria. Un mismo local con un profesorado único para las materias comunes y el exclusivo complemento de profesorado de cada especialidad.

Fue nombrado primer director, don Alfonso Barea y Molina. Más tarde pasó a ser Inspector de Primera Enseñanza del Protectorado. Secretario general don Manuel Chacón.

La escuela pasó por momentos críticos pero se pudieron solventar. Fue un día histórico para el Centro cuando se ordenó por el Directorio y por el Gobierno posterior, que la escuela, con todas sus secciones y subvenciones (el Estado, Instrucción Pública, Junta de Arbitrios) pasara a depender exclusivamente de la Dirección General de Marruecos y Colonias con el fin de establecer una conexión entre ésta y las Escuelas del Protectorado.

Las enseñanzas se agrupaban según el siguiente cuadro:

Cuatro secciones:

1.a) BACHILLERATO.-Se rige por disposiciones generales dadas por los Institutos Nacionales, con la plantilla que han de quedar los Institutos cuando se suma el turno de cátedras acumuladas.

2.8) NORMALES. - De maestros y maestras: profesorado el de la sección de Bachillerato para todas las asignaturas comunes con las tres secciones, completando con las siguientes enseñanzas especiales cada sección:

Pedagogía y su historia; Labores; Caligrafia; Música.

3. $\left.{ }^{a}\right)$ COMERCIO.-Dos cursos de Vulgarización; Derecho Mercantil y Geografia; Contabilidad; Aritmética y Cálculo mercantil; Taquigrafia y Mecanografía. 
4.) ARTES Y OFICIOS. TALLERES.-Metalistica; Modelado y Vaciado; Dibujo lineal y artistico.

\section{2.-La reforma del Instituto General y Técnico}

El cambio de nombre del Centro, denominado antes Escuela General y Técnica y a partir de estos momentos Instituto General y Técnico de Victoria Eugenia, fue en conmemoración de la visita de S.S. M.M., en la apertura del curso 19271928.

La reforma comprendía los siguientes capitulos:

\section{Capitulo Primero}

De las enseñanzas.

Articulo $10^{\circ}$ Secciones que comprenden:

Bachillerato; Magisterio Nacional; Enseñanzas de vulgarización; Enseñanzas industriales; Elementos de cultura para musulmanes; Enseñanza primaria.

La 1. Sección se imparte igual que en la península.

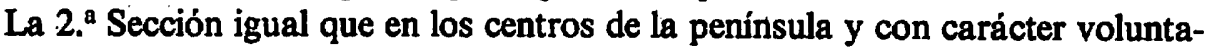
rio los siguientes:

Los cursos de Arabe vulgar o chelja a elección en el segundo y tercer año, y un curso de Derecho musulmán en el centro.

Los estudios de la 4. ${ }^{\mathrm{a}}$ Sección consistían en los de Vulgarización Mercantil, señalados en el artículo 66 del Real Decreto de 31 de agosto de 1922.

La 5. ${ }^{a}$ Sección comprende las materias que se integran en una Escuela de Aprendizaje siguiendo las normas del artículo 19 del Reglamento de Enseñanza Industrial de 6 de octubre de 1925.

6. ${ }^{\mathrm{a}}$ Sección: Elementos de cultura para musulmanes. Esta sección está destinada a aquellos indígenas que desean adquirir la preparación suficiente para desempeñar cargos auxiliares en los distintos ramos de la Administración del Protectorado o para ejercer profesiones libres y comprende dos clases de enseñanzas: cultura general y cultura profesional.

La primera consistirá en la enseñanza primaria española y será adquirida de la clase preliminar y en el Grupo escolar del instituto. La clase preliminar se formará con aquellos musulmanes que no poseyendo el idioma español no están en condiciones de aprovechar las enseñanzas del Grupo.

Las mejoras también afectaron a la Administración del Centro. Mejoras de emolumentos del personal docente. Distribución de las cantidades percibidas según categorías del personal docente y administrativo.

Las plazas debian ser cubiertas mediante un concurso-oposición.

Antes tenían validez en toda la península los estudios de Bachillerato, más no los de otras secciones. Ahora cesa esa anomalia.

Se coloca al Instituto en condiciones de atraer numeroso contingente de alum- 
nos de la ciudad, del campo y de Argelia.

\section{3.-La Escuela Elemental del Trabajo}

\subsection{1.-Lineas Generales}

Según la exposición del Decreto Ley de 31 de octubre de 1924, el Estado pretende elevar la capacidad productora del obrero impartiendo una enseñanza técnica. Elevando sus conocimientos técnicos se piensa salvar mucho de los efectos de crisis industriales.

Los principales males que vienen a remediar el Estado son el evitar que las enseñanzas industriales sostenidas por la Administración solo sirviesen de motivo para obtener subvenciones y nombramientos. Se refiere a las hasta ahora llamadas Escuelas de Artes y Oficios y que en lo sucesivo han de denominarse Escuelas Elementales del Trabajo o Escuelas de Aprendizaje, pues son éstas las únicas sostenidas por la Administración local a que alude el texto del referido Decreto Ley.

El artículo 17 del Estatuto (1924), previene, que cada Municipio con más de 20.000 habitantes queda obligado a sostener escuelas elementales, municipales, o subvencionar escuelas privadas inspeccionadas, capaces en total para una población escolar y minima de un alumno por cada 1.000 habitantes.

En Melilla existe la Escuela de Artes y Oficios incorporada a la Escuela General y Técnica y sostenida por la Junta de Arbitrios en la parte alicuota que le corresponde, de las 100.000 pesetas que anualmente consigna en sus presupuestos.

El artículo décimo del Real Decreto de 6 de octubre de 1925, determina que en el plazo de un mes, serán nombrados las Juntas locales donde se halle establecida alguna Escuela Elemental del Trabajo o donde los municipios, diputaciones, o mancomunidades, hayan consignado o deben consignar en sus presupuestos, las cantidades necesarias para aquel establecimiento.

\subsection{2.-Enseñanzas que se imparten}

La enseñanza elemental obrera comprende los tres grupos siguientes:

a) Enseñanzas preparatorias para el aprendizaje cuyo objeto es facilitar a los obreros que hubiesen cumplido 10 años, un complemento de instrucción científica y social que les capacite para el máximo aprovechamiento de las enseñanzas de aprendizaje; comprendurá dos cursos.

b) Enseñanzas de aprendizaje, encaminadas a la formación de buenos oficiales obreros; se imparten en cuatro años, con edad mínima de 12 años, reduciéndose la duración de los estudios a tres para los alumnos que hubieran cursado la enseñanza preparatoria. 
c) Cursos complementarios profesionales, destinados a los aprendices y obreros que trabajan durante todo el dia, los cuales deberán comprender, además de las enseñanzas propiamente técnicas, un complemento de cultura general cientifica y social apropiados a la capacidad de los alumnos.

En el periodo preparatorio para el aprendizaje se facilitarán las enseñanzas básicas comunes a los diversos oficios especializándose los estudios para cada oficio en el período de aprendizaje.

Los cursos complementarios representan un trabajo máximo de 12 horas semanales y se establecerán por las Juntas locales sometiendo el plan correspondiente a la aprobación del Ministerio.

\section{5.-La granja agricola de Melilla}

Un Real Decreto con fecha 25 de marzo de 1927 estableció enseñanzas prácticas de Agricultura en la Granja de Melilla y en el Campo de Experimentación de Larache, "para dar el mayor impulso posible a la agricultura de esta zona del Protectorado por medio de la difusión de los modernos métodos de explotación de las tierras, de la ganadería y de las industrias rurales".

El proyecto comprendia 14 artículos y establecían dos clases de enseñanzas; secundaria para los jóvenes marroquies de familias agricultoras, propietarios o cultivadores de tierras, y obrera, para obreros del campo, españoles o marroquies, que aspirasen a ser capataces agricolas. La enseñanza debería ser práctica y teórica. La primera abarcaba las principales operaciones de preparación de tierras, cuidados culturales a las plantas, recolección, selección de semillas, poda, injertos y plantaciones arbóreas, sericultura, avicultura y apicultura, medios para combatir las enfermedades de las plantas, reconocimientos de productos y primeras materias para la agricultura e industrias derivadas. Todo ello con una duración mínima de tres horas por día laborable.

La enseñanza teórica comprendía dos cursos en los que se impartian clases de Lengua española, Aritmética, Mineralogía, Agronomía, Botánica, Maquinarias agricolas, Zoología y Ganadería, Contabilidad agrícola, Elementos de industrias rurales y Patologia animal y vegetal.

La enseñanza obrera conduce, después del primer año, a una certificación acreditativa de la preparación adquirida y después del segundo, al título de Capataz Agrícola, que da derecho a ser destinado a las distintas secciones de las granjas y campos de experimentación y muy especialmente a las misiones de demostraciones prácticas ambulantes en campos y explotaciones rurales. Un Decreto, 22 de agosto de 1928, puso en vigor el Reglamento de Enseñanzas de Agriculturas, en estas Granjas-Escuelas, estableciendo que en la de Melilla, recibieran enseñanza secundaria jóvenes españoles y en la de Larache jóvenes marroquies, dándose la enseñaza para capataces en ambas. 


\section{6.-La enseñanza privada: los Hermanos de la Doctrina Cristiana}

Con Canalejas se votó la Ley de Bases del Ejército (29 de junio de 1911). Meses más tarde aparece la Ley de Reclutamiento. Los presbiteros estaban excentos, no así los religiosos. No obstante el artículo 238 recogia que los religiosos de las Congregaciones de Misiones reconocidas por actos oficiales durante la legislación anterior a 29 de junio de 1911 , prestaran como servicio militar, cuando les corresponda, el propio de su ministerio precisamente en las misiones españolas de Africa, Tierra Santa, América, Extremo Oriente y demás que el Gobierno determine. Se incluyen en el artículo cinco órdenes docentes: Escolapias, Hermanos de las Escuelas Cristianas, Marianistas, Maristas y Salesianos.

Partiendo de esta Ley, los Hermanos de la Doctrina Cristiana se establecieron en la Plaza de Melilla. Todo fueron facilidades por parte de las autoridades eclesiástica y no menos de los militares. Asi se abrió a la enseñanza privada el Colegio de Ntra. Sra. del Carmen el $10^{\circ}$ de septiembre de 1912.

Primo de Rivera estuvo a punto de suprimir el artículo 238, y sólo la intervención del marqués de Comillas logró hacerle desistir.

Aglutinó a toda la Enseñanza de Bachillerato hasta la creación de la Escuela General y Técnica (en el año 1922, de enseñanza pública).

Su labor no sólo fue un refugio para los hermanos llamados a filas, sino que rindió a Melilla servicios inestimables. En las aulas alternan cristianos, musulmanes y judios. La enseñanza era de pago.

Se abrieron las clases con Enseñanza Primaria y Comercio y en seguida la enseñanza de Bachillerato (por cierre del único colegio que lo imparte) a petición de gran número de militares.

Durante la Dictadura el ministro Eduardo Callejo, del primer Gobierno civil del general Primo de Rivera, llevó a cabo una reforma educativa: Ley de 1926 que reguló estudios, libros de texto, etc., y reconoció los centros superiores de la Iglesia. No obstante hay una oposición a los centros de la Iglesia. En 1927 La Verdad, publica los estatutos de la "Asociación Nacional de Enseñanza Privada".

En 1927 los Hermanos colaboran con la enseñanza pública y asumen la dirección de las escuelas gratuitas del "Ave Maria", que llegará hasta 1932.

\section{7.-Conclusiones}

En líneas generales podemos decir que durante el periodo de la Dictadura de Primo de Rivera, hubo una sensibilización a favor de la enseñanza en Melilla, que sobresalió durante el curso 1925-26, cuando éste se hace cargo de la Alta Comisaría y del mando militar de la Zona del Prctectorado Español de Marruecos.

La Junta de Arbitrios mantuvo una preocupación constante por las construcciones escolares. Sin embargo, no fue suficiente, puesto que la población infantil continuó hacinada y sin escolarizar en su totalidad. La primera enseñanza se duplicó en cuanto a profesorado y centros de enseñanza. Los maestros de ense- 
ñanza primaria consiguen que se les conceda el 50 por 100 sobre sus haberes, ya que eran los únicos funcionarios del Estado que no la disfrutaban.

A pesar de la legislación vigente y los manifiestos sobre la enseñanza faltó una mayor preocupación por parte del Gobierno en implantar la obligatoriedad de la enseñanza con carácter general, y los medios adecuados para llevarla a su cumplimiento.

La enseñanza media ascendió notablemente desde la creación de la Escuela General y Técnica (1921-1922), primer centro oficial de la segunda enseñanza. En ella observamos el desarrollo siguiente:

1. ${ }^{\circ}$ El crecimiento más importante de alumnado tiene lugar en la Sección de Bachillerato. Lo cual nos da idea de la necesidad que había en implantar esta enseñanza en nuestra ciudad. El mayor número de alumnos se da en el curso 1925-26. A partir del cual decrece notablemente.

2.) La Sección de Artes y Oficios triplica su matricula en el curso 1923-24. Hay que tener en cuenta las mejoras puestas en vigor por el Decreto Ley de 31 de octubre de 1924.

3. ) Las restantes secciones, Enseñanza Primaria, Magisterio y Comercio aumentan considerablemente durante el periodo $1924-25$ para sufrir un fuerte descenso y estancamiento a partir del curso 1926-27.

En cuanto a una especial diferenciación entre las enseñanzas pública y privada observamos que la enseñanza para los alumnos marroquies es totalmente gratuita en todos sus grados (primaria, media, especial y superior), en tanto que para los españoles es gratuita solamente la primaria. No sólo la gratuidad comprende el círculo de los estudios sino que se extiende para los necesitados, a la alimentación y ropa. Por contra, la enseñanza privada tuvo marcado carácter elitista, debido al elevado costo que suponia para la clase obrera, enfocada y fomentada por la Iglesia y la población militar existente. 